\title{
Assessment of quality of life in giant ameloblastoma adolescent patients who have had mandible defects reconstructed with a free fibula flap
}

Xiangru Li', Kuicheng Zhu' ${ }^{2}$ Fei Liu ${ }^{1}$ and Hongwen $\mathrm{Li}^{3^{*}}$

\begin{abstract}
Background: The reconstruction of mandibular defects after giant ameloblastoma resection is one of the most challenging problems facing reconstructive surgeons. Mandibular resection has been associated with a poor quality of life (QOL), particularly in adolescent patients reconstructed with a free fibula flap. This study aims to evaluate QOL outcomes in adolescent patients who have had mandibular resections of giant ameloblastoma and reconstruction with a free fibula flap and to collect information about their socio-cultural situation.
\end{abstract}

Methods: The present study assessed 45 adolescent patients who had undergone immediate mandible reconstruction with a free fibula flap for faint ameloblastoma using University of Washington Quality of Life (UW-QOL) and 14-item Oral Health Impact Profile (OHIP-14) questionnaires.

Results: Thirty-five of the 54 questionnaires were returned (64.81\%). In the UW-QOL, of the twelve disease-specific domains, the best three scores from the patients related to pain, shoulder and appearance and the worst three scores related to chewing, anxiety and saliva. In the OHIP-14, the lowest-scoring domain was handicap, followed by physical pain and social disability.

Conclusions: Mandibular reconstruction with a free fibular flap significantly influenced the adolescent patients' QOL. Adolescent patients pay more attention to postoperative facial appearance; this should be considered in surgical planning.

Keywords: QOL, Free fibula flap, Ameloblastoma, Reconstruction

\section{Background}

In the recent histologic classification of odontogenic tumors from the World Health Organization (WHO), ameloblastoma is defined as a benign, locally invasive epithelial odontogenic neoplasm of putative enamel organ origin. Ameloblastomas are benign, locally-invasive odontogenic neoplasms of epithelial origin, which are derived from the tooth-forming apparatus [1]. They account for approximately $60 \%$ of odontogenic tumors of the mandible and $10 \%$ of all jaw tumors [2]. The tumor was first described by Broca in 1868 and named by Churchill. Although it is

\footnotetext{
* Correspondence: zzyfykq4@163.com

${ }^{3}$ Dermatology, the First Affiliated Hospital of Zhengzhou University,

Zhengzhou university, Zhengzhou, Henan 450052, China

Full list of author information is available at the end of the article
}

considered to be benign, it may show various biological behaviors ranging from cystic expansion to a more aggressive solid mass and even malignant transformation.

Ameloblastomas often present as painless, slow-growing tumors that cause expansion resulting in perforation of the cortical bone and eventually infiltrate into the adjacent soft tissue. Approximately 10 to $15 \%$ of all ameloblastoma occur in young patients; it is considered a rare tumor in these groups $[3,4]$.

In recent years, radical resection and immediate reconstruction have become the state-of-the-art procedures in the management of ameloblastoma. Fibula is the free flap most often used to manage extensive ameloblastoma of the mandible because it aims to provide 
adequate cosmetic and functional results. In 1989, Hidalgo reported a significant series of free fibula flaps for mandibular reconstruction [5]. The free vascularized fibula flap provides the longest segment of bone, with 20 to $30 \mathrm{~cm}$ available for harvest. This flap can be used to span an angle-to-angle defect. The bone is also of adequate width and height to allow for placement of osseointegrated dental implants [6,7].

Mandibular reconstruction after resection of a tumor is extremely important as it improves the patient's quality of life (QOL). Mandibular function and facial aesthetics are aspects that must be preserved [8]. Assessment of the QOL provides information about the psychosocial well-being of patients and the effects of the disease and its treatment. Hence, it is an important tool for evaluating outcome in conjunction with mortality, morbidity, survival and recurrence rates. QOL has become an increasingly important outcome measure for patient's undergoing treatment for a wide array of illnesses.

The aim of our study was to evaluate by questionnaire the QOL of adolescent patients who have had mandibular defects after trauma or tumor resection and reconstructions with a free fibula flap. To our knowledge, few studies have considered this in relation to adolescent patients' free fibula flap reconstruction. The findings could potentially provide useful information for physicians and ameloblastoma patients while treatment is being planned.

\section{Method}

\section{Details of patients}

Our study was granted an exemption in writing by the Ethical Review Board of the First Affiliated Hospital of Zhengzhou University. There were 165 cases of ameloblastoma from all age groups diagnosed in the Department of Stomatology at the First Affiliated Hospital of Zhengzhou University from March 2004 to March 2013. Fifty-four patients from this group who were diagnosed by histopathology with giant ameloblastoma, and who underwent immediate mandibular reconstruction with a free fibula flap, were adolescent patients and are included in this study.

They all satisfied the inclusion criteria: the free fibular flap survived completely; there were no severe postoperative complications; they had no previous or synchronous malignancies and no cognitive impairment; and they were at least 12 months after reconstruction and aged less than 24 years.

Fifty-four patients (41 men, 13 women) met the inclusion criteria. Most adolescent patients completed the questionnaire when they returned to the hospital for review. The remaining patients received a formal letter explaining the study, an informed consent form, and the University of Washington Quality of Life (UW-QOL) and the 14-item Oral Health Impact Profile (OHIP-14) questionnaires, as well as a questionnaire exploring social and educational status. Patients who did not reply within one month received a reminder. Patients, who could not fill in the questionnaires themselves, for example because of dementia or language problems, were excluded from the study.

We compiled and reviewed data for preoperative variables (tumor primary site, sex, age); reconstruction flaps characteristics (flap complications and flap success); followup information (recurrence and survival situation).

\section{Questionnaires and data collection}

Although many generic QOL instruments have been developed over the past 30 years, the OHIP-14 [9] and the UW-QOL [10] are most commonly used for cancer patients. The UW-QOL scale is filled in by the patient and provides a broad measure of QOL for patients with head and neck cancer with good acceptability, practicality, validity, reliability, and responsiveness [11]. The questionnaire is composed of 15 domains: 12 are disease-specific items (pain, appearance, activity, recreation, swallowing, chewing, speech, shoulder, taste, saliva, mood, and anxiety), and 3 are global questions. Each of the twelve included questions has three to six response options. The domains are scored on a scale ranging from 0 (worst) to 100 (best). Besides the 15 questions, patients were asked to choose no more than 3 of the 12 disease-specific domains that had been the most important to them in the preceding 7 days. We scored the individual domains according to the UWQOL guidelines. The standard UW-QOL is available as a Chinese version and has been validated for a Chinese population [12].

The OHIP-14 consists of 14 items divided into 7 different domains: functional limitation, physical pain, psychological discomfort, physical disability, psychological disability, social disability, and handicap. Each item is scored as: $0=$ never; 1 = hardly ever; 2 = sometimes; 3 = fairly often; and $4=$ very often. The domains are scored on a scale ranging from 0 (best) to 100 (worst). The higher the score, the poorer the patient's state of health. The standard OHIP-14 is available as a Chinese version and has been validated for a Chinese population [12].

\section{Statistical analysis}

Data were recorded and analyzed with SPSS 16.0 statistical software (SPSS Inc., Chicago, IL, USA). The significance level was set at $P<0.05$.

\section{Result}

\section{Patients}

Of the 54 patients who were sent a questionnaire, 35 (64.81\%) completed and returned it. Eight questionnaires were returned stating that the patients had changed their address, six patients declined to participate, and five 
claimed not to have received the questionnaire or the reminder. Of the 35 patients who completed the questionnaires there were 23 men and 12 women, median age 17 (range 10 to 24). The postoperative follow-up period ranged from 12 to 32 months. Details of the patients and their tumors are shown in Table 1.

There were $14(40.0 \%)$ tumors on the left side, 12 (34.28\%) on the right, and $9(25.72 \%)$ that crossed the midline. There were $15(42.86 \%)$ tumors in the body and angle of the mandible, $12(34.28 \%)$ that occupied the body-angle-ramus of the mandible, $8(22.86 \%)$ in the body of the mandible. Sizes of the lesions ranged from $3.8 \times$ $1.3 \mathrm{~cm}^{2}$ to $6.4 \times 4.3 \mathrm{~cm}^{2}$. Radiographic appearance in 24 (68.57\%) cases was unilocular; $11(31.43 \%)$ cases were multilocular. Clinical classification included 26 (74.29\%) solid type and 9 (25.71\%) unicystic type tumors.

\section{Quality of life}

UW-QOL: the scores for 12 disease-specific domains and the importance of each domain are all shown in Table 2. Topping the list of GOOD-scoring domains was pain (82.21 \pm 5.78$), 42.85 \%$ of patients scoring $\geq 80 \%$ in pain domain. Of the twelve disease-specific domains, the best three scores were for pain, shoulder and appearance; the worst three scores were for chewing, anxiety and saliva. Chewing was considered to be the most important issue in the previous seven days, followed by speech and appearance.

Importance of domains: percentage of patients that considered that particular domain important (up to three domains per patient).

OHIP-14: distributions of OHIP-14 domain scores at presentation are shown in Table 3 . The best domain scores

\section{Table 1 Patient profiles}

\begin{tabular}{lll}
\hline Variables & N & $\%$ \\
\hline Age & 26 & $74.29 \%$ \\
$<18$ years & 9 & $25.71 \%$ \\
$\quad 18$ to 24 years & & \\
Gender & 23 & $65.71 \%$ \\
$\quad$ Male & 12 & $34.29 \%$ \\
$\quad$ Female & & \\
Primary tumor sites & 15 & $42.86 \%$ \\
$\quad$ Body-angle & 12 & $34.28 \%$ \\
$\quad$ Body-angle-ramus & 8 & $22.86 \%$ \\
$\quad$ Body & & $74.29 \%$ \\
Clinical type & 26 & $25.71 \%$ \\
Solid & 9 & \\
$\quad$ Unicystic & & $31.43 \%$ \\
Radiographic appearance & & \\
$\quad$ Unilocular & 24 & \\
Multilocular & 11 &
\end{tabular}

for the complete group were 36.34 for handicap, 38.54 for physical pain, and 40.24 for social disability. The highest score was for psychological disability $(53.45 \pm 2.54)$ and physical disability $(73.14 \pm 10.24)$. No patients scored $40 \%$ or less in physical disability.

There were a total of twenty-six (74.29\%) adolescent patients in school. Four (11.42\%) patients in primary education. Seven patients $(20.00 \%)$ in junior middle school, nine (25.71\%) patients in senior school, six patients $(17.14 \%)$ in university graduation. Four patients could not read or write, and needed parents help to complete the questionnaire.

\section{Discussion}

Ameloblastomas are benign, locally-invasive odontogenic neoplasms of epithelial origin, which are derived from the tooth-forming apparatus. As the name implies, it was assumed that ameloblastomas are derived from ameloblasts. This assumption has now almost certainly been confirmed in studies that have shown that the cells of origin are epithelial cells that can express amelogenin [13]. The treatment of giant ameloblastoma tumors affecting the jaw bones often requires extensive resection and results in loss of bone continuity, modifying the contour of the face. Mandibular resection often involves the loss of an important bone segment, including teeth. Mandibular bone defects can cause asymmetry, facial disharmony, and tooth loss which compromises chewing. The mandible plays a major role in airway protection and support of the tongue, lower dentition, and the muscles of the floor of the mouth permitting mastication, articulation, deglutition, and respiration. It also defines the contour of the lower third of the face. Interruption of mandibular continuity, therefore, produces both a cosmetic and functional deformity [14].

The free fibula flap as a source of vascularized bone in reconstructive surgery is in wide use. The fibula has been demonstrated to be an ideal flap for mandibular reconstruction. This is particularly true when a limited number of fibular osteotomies are needed to provide appropriate bone shape. When the needs of reconstruction involve muscle bulk, the flexor hallucis longus can be incorporated in the flap. Because the craniofacial bones, teeth, and soft tissues of adolescent are not completely developed, surgery has the potential to cause irreversible deformities, presenting a great challenge for doctors, who must not only treat the disease, but also consider the aesthetic results in light of the postoperative growth and development of these adolescent patients.

The expectation of clinical outcome of reconstruction is regarded to be the most important factor in the decision, and QOL measurement provides information about perceptions of patients [15]. The relatively large number of questionnaires specific for diseases of the oral cavity reflects the fact that there is no 'gold standard'. We used 
Table 2 Means of scores of items and scales of UW-QOL questionnaire

\begin{tabular}{|c|c|c|c|c|c|c|c|}
\hline & Mean & SD & Median & Range & GOOD score, \%* & Importance of domains, \%* & Rank order \\
\hline \multicolumn{8}{|l|}{ UW-QOL } \\
\hline Pain & 82.21 & 5.78 & 82.00 & 60 to 93 & $42.85 \%$ & $0 \%$ & 11 \\
\hline Appearance & 78.12 & 11.56 & 78.00 & 60 to 90 & $25.71 \%$ & $48.57 \%$ & 3 \\
\hline Activity & 69.48 & 7.56 & 69.00 & 40 to 84 & $8.57 \%$ & $17.14 \%$ & 7 \\
\hline Recreation & 68.21 & 10.59 & 68.00 & 50 to 78 & $0 \%$ & $14.29 \%$ & 8 \\
\hline Swallowing & 77.32 & 6.77 & 77.00 & 45 to 88 & $28.57 \%$ & $5.71 \%$ & 10 \\
\hline Chewing & 28.48 & 3.18 & 29.00 & 0 to 68 & $0 \%$ & $77.14 \%$ & 1 \\
\hline Speech & 71.26 & 12.57 & 71.00 & 35 to 87 & $22.86 \%$ & $54.29 \%$ & 2 \\
\hline Shoulder & 80.29 & 9.01 & 81.00 & 55 to 88 & $40.0 \%$ & $0 \%$ & 11 \\
\hline Taste & 71.23 & 8.76 & 71.00 & 52 to 88 & $25.71 \%$ & $11.43 \%$ & 9 \\
\hline Saliva & 60.02 & 7.62 & 60.00 & 30 to 82 & $42.42 \%$ & $22.86 \%$ & 5 \\
\hline Mood & 67.09 & 1.15 & 68.00 & 30 to 90 & $11.42 \%$ & $20.00 \%$ & 6 \\
\hline Anxiety & 55.76 & 8.23 & 55.00 & 26 to 70 & $0 \%$ & $28.57 \%$ & 4 \\
\hline
\end{tabular}

${ }^{*}$ GOOD score $\%: \%$ scoring $\geq 80$.

the OHIP-14 and UW-QOL questionnaires to assess the postoperative QOL of adolescent patients and the possible relation to reconstructive surgery with a free fibula flap.

The oral specific questionnaire was able to better demonstrate the changes in quality of life due to surgery. Many scholars have chosen to use the UW-QOL questionnaire. The UW-QOL measure was chosen as the head and neck specific questionnaire because it is short and easy for patients to complete themselves, thus making it ideal in a busy outpatient setting. We can see that the highest score of UW-QOL subscales in this study was in the pain domain $(82.21 \pm 5.78), 42.85 \%$ of patients scoring $\geq 80 \%$ in this domain, which indicated a slight damage in the pain domain. Of the twelve disease-specific domains, the best three scores were for pain, shoulder and appearance; the worst three scores were for chewing, anxiety and mood. A remarkable finding was that the high score of UW-QOL subscales in this study was in the pain domain. The mean score was 82.21 , which indicated a great improvement in pain. We found anxiety (55.76 \pm $8.23)$ and mood $(67.09 \pm 1.15)$ domains to have a low score. This suggested that some adolescent patients were in a bad psychological state and that the surgery had some negative influence.

The appearance domain of UW-QOL had been identified as a predictor of the overall burden of oral cancer patients. We found that $48.57 \%$ patients were satisfied with the appearance domain. This may be due to free fibula flaps providing us with the opportunity to more carefully address the aesthetic and functional reconstruction of mandible defects based on the wide variety of bone and soft tissue available. The reconstruction of the mandible retained the contour of the lower third of the face, thus, giving patients a better postoperative facial appearance.

However, a remarkable finding was that the lowest score of UW-QOL was in the domain of chewing. This may be because mandibular defects cause the loss of some teeth and, therefore, the ability to chew. This confirms the report of Rogers et al. [16] who showed that patients had chewing problems after segmental mandibulectomy and reconstruction using a composite free tissue transfer. Dental implants can resolve this problem, but are difficult in Chinese patients because immediate dental implant placement prolongs rehabilitation time, increases the number of required surgical procedures and imposes an additional economic burden. However, we believe that dental implant placement should be considered an integral part of treatment, especially in adolescent patients.

Hsing et al. and Rogers et al., in their studies on importance-rating using the UW-QOL questionnaire in patients treated by primary surgery for oral cancer, found that they tended to rate speech, chewing, and swallowing as more important than the other UW-QOL domains. Other studies of importance-rating include Lin et al. whose results emphasised swallowing, chewing and speech as important and $\mathrm{Li}$ et al. whose results rated chewing, swallowing, and saliva $[11,12,14]$ as important. Wang et al. in their study on importance-rating used the UW-QOL in similar patients to ours and found that patients tended to rate swallowing, chewing, and speech as more important than the other UW-QOL domains [15]. We found that chewing, speech, and appearance were the most important. This is maybe because in traditional Chinese culture we pay more attention to diet than to other domains. 
The OHIP-14 was designed to provide a comprehensive measure of the dysfunction, discomfort, and disability attributed to oral conditions. The OHIP-14 consists of 14 items organized into 7 subscales that assess how oral health can affect physical and social wellbeing. In addition the patient can complete it in ten minutes. There were some lower score in the domains of Handicap (36.34 \pm $0.95)$, Physical Pain $(38.54 \pm 1.11)$ and Social disability (40.24 \pm 10.24$)$; however, postoperatively there were higher scores for $(73.14 \pm 10.24)$ and Physical Disability (53.45 \pm 754). No patients scored $40 \%$ or less in Physical Disability, which shows that operations for mandibular resections of giant ameloblastoma in adolescent patients and reconstruction with a free fibula flap do have an overall effect on oral health.

Twenty-six (74.29\%) adolescent patients in school. Four $(11.42 \%)$ patients in primary education. Seven patients (20.00\%) in junior middle school, nine (25.71\%) patients in senior school, six patients $(17.14 \%)$ in university graduation. Four patients could not read or write, and needed parents help to complete the questionnaire. For some adolescent patients, physical and psychological immaturity made illness interpretation very difficult for doctors and so parents needed to assist by giving the correct interpretation of their child's illness. The UW-QOL and OHIP-14 do not contain a section on the effect of the free fibula flap donor site on QOL and function. The free fibula flap's primary donor site left a scar, but as the donor site closure is hidden, patients can easily accept donor site morbidity. In our study, some patients were worried about moving the operated limb, thereby reducing the normal activities. However, Farhadi et al. found no clinically obvious difference in movement between the donor and un-operated limbs [15]. We compared the patients' donor and unoperated control legs with regard to range of motion of ankle inversion and eversion and normal leg movements. All patients showed that there were no significant differences between the two leg movements after 12 months.

The radiographic appearance of ameloblastoma in adolescents can be classified as unilocular or multilocular. In studies by Kahn et al. [17] and Huang et al. [18], the majority of tumors were unilocular $(68.42 \%$ and $66.67 \%$, respectively). This was also true of the present study, in which $68.57 \%(24 / 35)$ of the patients had unilocular lesions. Most authors have reported that [19] unilocular tumors have more complete cystic walls with weak local infiltration, a small degree of bone involvement, and no residual tumor after thorough curettage. However, multilocular tumors are highly destructive and invasive, and conservative treatment often leaves residual tumor, frequently recurrent.

Because the craniofacial bones, teeth, and soft tissues of adolescents are not completely developed, surgery has the potential to cause irreversible deformities, presenting a great challenge for doctors, who must not only to treat the disease, but also consider the aesthetic results in light of the postoperative growth and development of these adolescent patients.

There were some limitations in our study. First, this was not a randomized study. Selection bias inevitably existed. Second, although the treatment guidelines are standardized at the study institute, individual variations among surgeons certainly exist. Finally, the time from treatment to questionnaire was not uniform for each patient.

\section{Conclusions}

Immediate mandibular reconstruction with a free fibula flap for giant ameloblastoma patients would have significantly influenced the adolescent patients' quality of life, especially regarding their chewing, anxiety levels and mental health. Adolescent patients pay more attention to appearance and activity domains than other age groups, so this should be considered for surgical planning.

\section{Competing interests}

The authors declare that they have no competing interests.

\section{Authors' contributions}

$\mathrm{XL}, \mathrm{KZ}$ and $\mathrm{FL}$ participated in the clinical management of the patient and wrote the manuscript. HL were involved in the final editing. All authors read and approved the final manuscript.

\section{Author details}

'Department of Stomatology, the First Affiliated Hospital of Zhengzhou University, Zhengzhou University, Zhengzhou 450052, Henan, China. 'Laboratory Animal Center, Zhengzhou University, Zhengzhou 450052, Henan, China. ${ }^{3}$ Dermatology, the First Affiliated Hospital of Zhengzhou University, Zhengzhou university, Zhengzhou, Henan 450052, China.

Received: 11 February 2014 Accepted: 29 June 2014

Published: 8 July 2014

\section{References}

1. Snead ML, Luo W, Hsu DD, Melrose R, Lau EC, Stenman G: Human ameloblastoma tumors express the amelogenin gene. Oral Surg Oral Med Oral Pathol 1992, 74:64-72.

2. Olaitan AA, Adekeye EO: Clinical features and management of ameloblastoma of the mandible in children and adolescents. $\mathrm{Br} J \mathrm{Oral}$ Maxillofac Surg 1996, 34:248-251.

3. Ghandhi D, Ayoub AF, Pogrel MA, MacDonald G, Brocklebank LM, Moos KF: Ameloblastoma: a surgeon's dilemma. J Oral Maxillofac Surg 2006, 64:1010-1014

4. Li W, Liu F, Zhongfei X, Huang S, Zhu W, Sun C: Treatment of ameloblastoma in children and adolescents. J Hard Tissue Biol 2012, 21:121-126

5. Hidalgo DA: Fibula free-flap: a new method of mandible reconstruction. Plast Reconstr Surg 1989, 84:71-79.

6. Cordeiro PG, Disa JJ, Hidalgo DA, Hu QY: Reconstruction of the mandible with osseous free flaps: a 10-year experience with 150 consecutive patients. Plast Reconstr Surg 1999, 104:1314-1320.

7. Chang YM, Wallace CG, Tsai CY, Shen YF, Hsu YM, Wei FC: Dental implant outcome after primary implantation into double-barreled fibula osteoseptocutaneous free flap-reconstructed mandible. Plast Reconstr Surg 2011, 128:1220-1228.

8. Murphy BA, Ridner S, Wells N, Dietrich M: Quality of life research in head and neck cancer: a review of the current state of the science. Crit Rev Oncol Hematol 2007, 62:251-267.

9. Slade GD, Spencer AJ: Development and evaluation of the Oral Health Impact Profile. Community Dent Health 1994, 11:3-11. 
10. Rogers SN, Lowe D, Fisher SE, Brown JS, Vaughan ED: Health-related quality of life and clinical function after primary surgery for oral cancer. Br J Oral Maxillofac Surg 2002, 40:11-18.

11. Rogers SN, Gwane S, Lowe D, Humphris G, Yueh B, Weymuller EA: The addition of mood and anxiety domains to the University of Washington quality of life scale. Head Neck 2002, 24:521-529.

12. Li W, Yang Y, Xu Z, Liu F, Cheng Y, Xu L, Changfu S: Assessment of quality of life of patients with oral cavity cancer who have had defects reconstructed with free anterolateral thigh perforator flaps. $\mathrm{Br} J \mathrm{Oral}$ Maxillofac Surg 2013, 51:497-501.

13. Kumamoto H, Yoshida M, Ooya K: Immunohistochemical detection of amelogenin and cytokeratin 19 in epithelial odontogenic tumors. Oral Dis 2001, 7:171-176.

14. Wang L, Su YX, Liao GQ: Quality of life in osteoradionecrosis patients after mandible primary reconstruction with free fibula flap. Oral Surg Oral Med Oral Pathol Oral Radiol Endod 2009, 108:162-168.

15. Farhadi J, Valderrabano V, Kunz C, Kern R, Hinterman B, Pierer G: Free fibula donor-site morbidity: clinical and biomechanical analysis. Ann Plast Surg 2007, 58:405-410.

16. Rogers SN, Devine J, Lowe D, Shokar P, Brown JS, Vaugman ED: Longitudinal health-related quality of life after mandibular resection for oral cancer: a comparison between rim and segment. Head Neck 2004, 26:54-62.

17. Huang IY, Lai ST, Chen $\mathrm{CH}$, Chen CM, Wu CW, Shen YH: Surgical management of ameloblastoma in children. Oral Surg Oral Med Oral Pathol Oral Radiol Endod 2007, 104:478-485.

18. Kahn MA: Ameloblastoma in young persons: a clinicopathologic analysis and etiologic investigation. Oral Surg Oral Med Oral Pathol 1989, 67:706-715.

19. Altini M, Coleman H, Kieser J, Kola H, Sneider P: Three-dimensional computed tomography reconstruction in treatment planning for large ameloblastoma. Ora Surg Oral Med Oral Pathol Oral Radiol Endod 1996, 81:619-622.

doi:10.1186/1477-7819-12-201

Cite this article as: Li et al: Assessment of quality of life in giant ameloblastoma adolescent patients who have had mandible defects reconstructed with a free fibula flap. World Journal of Surgical Oncology 2014 12:201.

\section{Submit your next manuscript to BioMed Central and take full advantage of:}

- Convenient online submission

- Thorough peer review

- No space constraints or color figure charges

- Immediate publication on acceptance

- Inclusion in PubMed, CAS, Scopus and Google Scholar

- Research which is freely available for redistribution 